The effect of droplet ejection frequency on the dimensions of inkjet-etched micro-via holes in poly4(-vinyl phenol) thin films

This article has been downloaded from IOPscience. Please scroll down to see the full text article.

2012 J. Phys. D: Appl. Phys. 45125303

(http://iopscience.iop.org/0022-3727/45/12/125303)

View the table of contents for this issue, or go to the journal homepage for more

Download details:

IP Address: 131.231.136.78

The article was downloaded on 06/03/2012 at $14: 32$

Please note that terms and conditions apply. 


\title{
The effect of droplet ejection frequency on the dimensions of inkjet-etched micro-via holes in poly4(-vinyl phenol) thin films
}

\author{
Yan Zhang, Changqing Liu and David C Whalley \\ Wolfson School of Mechanical and Manufacturing Engineering, Loughborough University, \\ Loughborough, Leicestershire, LE11 3TU, UK \\ E-mail: C.Liu@lboro.ac.uk
}

Received 4 November 2011, in final form 12 January 2012

Published 6 March 2012

Online at stacks.iop.org/JPhysD/45/125303

\begin{abstract}
The relationship between the size of inkjet-etched via-holes produced in poly4(-vinyl phenol) thin films and the number of ethanol drops dispensed was established for a range of droplet ejection frequencies. The physical mechanism underlying this relationship is proposed and the dependence of the development of via-hole dimensions on the droplet ejection frequency is believed to be attributable to the extent of evaporation of the solvent between two consecutive drop dispenses. The results indicate complete penetration of the via-holes through the polymer layer when produced at low droplet ejection frequencies. Electroplating was used to deposit $\mathrm{Cu}$ into the created holes to further confirm the absence of polymer residue at low frequencies. A threshold frequency, under which no via-hole enlargement occurs, has been found. The study systematically focuses on the influence of droplet ejection frequency on the size of the inkjet-etched via-holes versus the number of droplets used in poly4(-vinyl phenol) dielectric thin films for printed electronics application.
\end{abstract}

(Some figures may appear in colour only in the online journal)

\section{Introduction}

Inkjet printing has demonstrated its advantages in additive manufacturing over the past decade as a noncontact, maskless and material-saving process. Selective deposition of various functional materials, micro-structures and components by inkjet printing has become attractive for the manufacture of printed electronic components and for system integration [1-6]. Meanwhile, miniaturization of integrated circuits continues to be the driver in traditional Si-based electronics to achieve better performance at lower cost. These same drivers create a demand for technologies that allow 3D interconnections in printed electronics. This requires via-holes to be created through multiple dielectric layers for subsequent filling with an interconnect material. The dielectric layers are typically polymeric and one technique whereby vias can be formed in them is by locally dissolving the polymer using inkjet printed solvent droplets. Inkjet printers nowadays can be assembled with arrays of nozzles to hold different types of ink for different functional purposes. This technique can facilitate system integration based entirely on inkjet printing and simplify manufacturing procedures.

It is well known that the mass transfer from the centre to the periphery within a sessile drop on a surface during solvent evaporation can result in the transport and then precipitation at the periphery of material that was previously uniformly dispersed in the solvent. This phenomenon is known as the coffee stain effect or the coffee ring effect, and it is normally preferred to minimize this effect during applications of inkjet printing, such as conductive track printing, as the electrical performance can be affected by uneven track thicknesses. Nonetheless, this phenomenon can be used for deliberately modulating printed structures for many applications. For example, it has been demonstrated that it can be used to fabricate concave and convex microlenses $[7,8]$ and light-emitting diodes (LEDs) [9] that can be used in optoelectronics. Studies on using inkjet or other dispensing tools to define structures in polymers and the influence of factors on the appearance and dimensions of the generated features have also been carried out. Li et al [10] reported 
on the dependence on the polymer molar mass of polymer structures swollen by solvent drops. Karabasheva et al [11] and Grimaldi et al $[12,13]$ investigated the effect of solvent mixtures on the generated structures in polymer substrates. Kawase et al [14] first demonstrated making via-holes for interlayer communication in printed electronics and found that the diameter of via-holes produced remained unchanged despite increasing the number of droplets. However, de Gans et al [15] discovered that the diameter of the via-holes they produced obeyed a power law relationship with an increasing number of dispensed drops. de Gans et al used a droplet ejection frequency of $200 \mathrm{~Hz}$, but Kawase et al did not specify the frequency they used. However, it can be inferred that a low frequency was employed, which may explain these contrasting observations. Impacts of other parameters on the dimensions and appearance of the generated pattern in different polymer/solvent or particles/solvent systems have also been investigated [16-23]. Nonetheless, few studies focus on the interrelationship between droplet ejection frequency, the number of drops and the resulting via-hole dimensions, which is significant in studying this inkjet-etching via-hole formation technique and its feasibility for micro-via production in printed electronics applications.

Although this method of via-hole formation relies on the wetting and evaporation characteristics of the droplet on the polymer substrate and the rate of dissolution of polymer into the solvent, the size of the via-hole is primarily determined by the volume of the droplet impacting on the substrate [14,24]. Accordingly, one can expect that the droplet ejection frequency will influence the diameter of the via-holes created if the frequency is high enough for incomplete droplet evaporation to occur between impacts. In addition, Xia et al $[9,25]$ detected a thin residual layer several nanometres thick at the centre of the via-hole, which indicated that the hole was not completely etched through to the bottom. This may cause an electrical connection failure after such a via-hole is filled with a conductive material. However, it has been shown that repeated solvent dispensing will eventually transfer this residue away from the bottom of the hole [9]. Therefore, the size development of the via-hole with the number of drops at different drop ejection frequencies is of great interest.

\section{Experimental procedure}

Poly4(-vinyl phenol) powder with a molecular weight of 11000 and with $\leqslant 0.2 \mathrm{wt} \%$ of monomer, was dissolved into isopropyl alcohol in an ultrasonic bath to break up any agglomeration so that a clear yellow-coloured solution was formed. Standard $76 \mathrm{~mm} \times 51 \mathrm{~mm}$ glass microscopy slides were used as the substrates onto which a thin layer of the poly4(-vinyl phenol) solution was applied by spin coating at $1500 \mathrm{rpm}$ for $30 \mathrm{~s}$. The layer thickness was controlled by varying the solution concentration. A chromatic length aberration gauge was utilized to measure the thickness of the polymer layer.

For this work, anhydrous ethanol (200 proof) was used as the etchant and a drop-on-demand inkjet printer from Microfab was utilized to dispense ethanol drops. The nozzle diameter

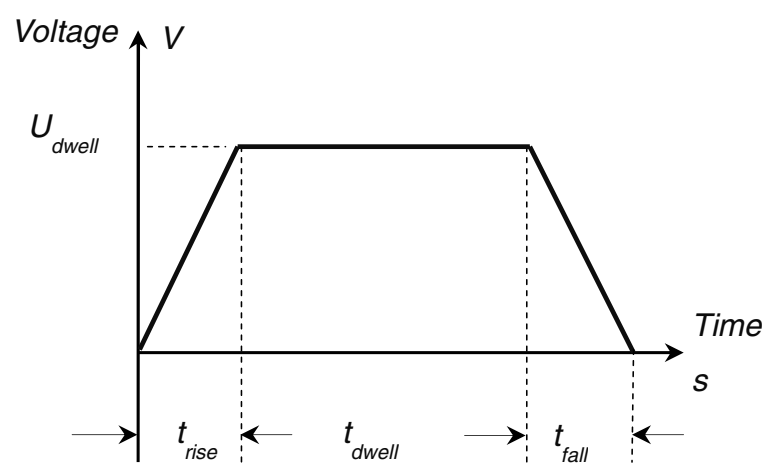

Figure 1. The unipolar waveform used to trigger jetting.

was $60 \mu \mathrm{m}$. The inkjet printer was set to the 'single' mode, where a signal applied to the jetting head triggers ejection of a single droplet. A unipolar waveform was employed to trigger droplet ejection, as shown in figure 1. This waveform is the voltage applied to the piezoelectric transducer within the jetting head. Both bipolar and unipolar waveforms are available on the Microfab Jetlab 4 inkjet printer; however, unipolar waveforms were used in this experiment to simplify the parameter adjusting process. The jetting parameters were optimized so that the ethanol was jetted without satellite drops and so that the trajectory of the drops was as perpendicular to the substrate as possible so as to minimize deviation of the droplet impact location on the substrate. This ensures that the droplet volume is constant and the drops land at the same location in the polymer layer. A script was compiled to print the required number of drops at specific coordinates on the printing plane. The profiles and dimensions of the resulting via-holes were obtained using a white-light interference microscope.

\section{Results and discussion}

\subsection{Development of via-hole dimensions at different droplet ejection frequencies $(f)$}

The concentration of the polymer/solvent solution used in these experiments was $11.3 \mathrm{wt} \%$, resulting in a polymer layer thickness of approximately $1.27 \mu \mathrm{m}$. The jetting parameters were $t_{\text {rise }}=t_{\text {fall }}=3 \mu \mathrm{s}, t_{\text {dwell }}=35 \mu \mathrm{s}$ and $U_{\text {dwell }}=24 \mathrm{~V}$, resulting in a droplet diameter of approximately $40 \mu \mathrm{m}$, which equates to a droplet volume of $31.4 \mathrm{pl}$. It is known that the droplet diameter is usually approximately the size of the nozzle diameter; however, it has been reported that jetting parameters can influence the diameter of the jetted droplet [26,27]. The smaller droplet diameter compared with the nozzle achieved here is attributed to the absence of satellite tails behind the main drop, which usually merge into the main drop and enlarge its volume. Due to the Newtonian behaviour of ethanol, fine tuning of the waveform eliminates the satellite formation. The dispensing frequency was varied between $1 \mathrm{~Hz}$ and $50 \mathrm{~Hz}$, and for each frequency, 23 via-holes were produced. The first 20 via-holes were created by 1-20 drops, respectively, and the last three were produced by 30,40 and 50 drops, respectively. Printing using such a pattern gives a detailed picture of the development of diameter versus the number of drops dispensed 


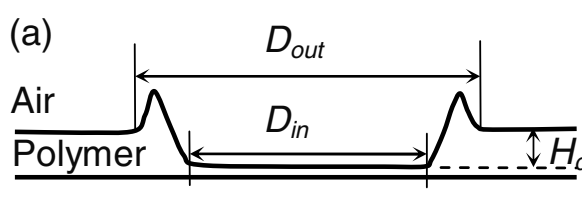

Substrate

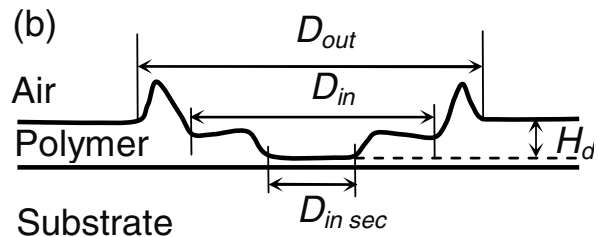

Figure 2. The profile of $(a)$ a typical inkjet-etched via-hole, $(b)$ a via-hole with a secondary hole at the bottom.
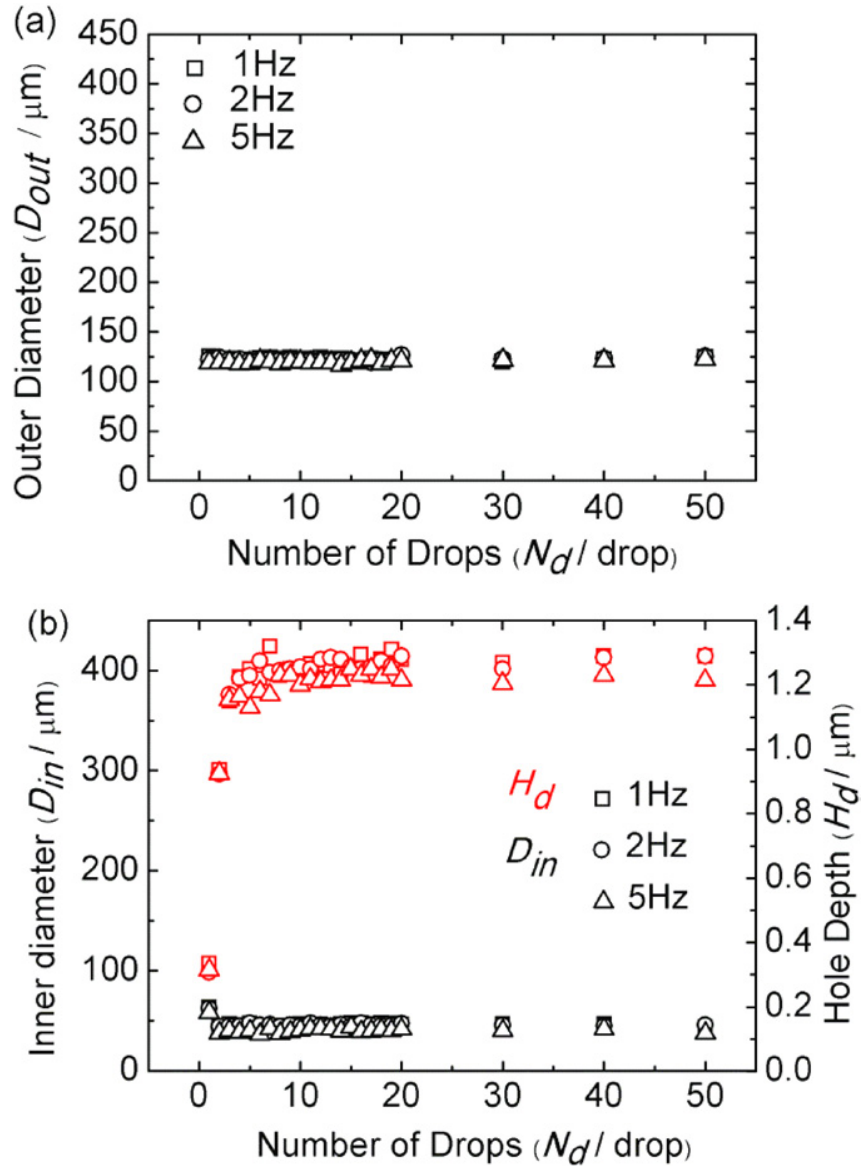

Figure 3. The profile of (a) $D_{\text {out }}$ versus $N_{\mathrm{d}}(b) D_{\text {in }}$ and $H_{\mathrm{d}}$ versus $N_{\mathrm{d}}$ at 1,2 and $5 \mathrm{~Hz}$.

during the initial stages while avoiding unnecessary repetition for larger numbers of droplets. All experiments were carried out in the ambient atmosphere.

Figure 2(a) schematically presents the typical profile of an inkjet-etched via-hole in the polymer layer and the definition of its dimensions: outer diameter $\left(D_{\text {out }}\right)$, inner diameter $\left(D_{\text {in }}\right)$ and via-hole depth $\left(H_{\mathrm{d}}\right)$. Figure $2(b)$ demonstrates the profile of a via-hole with a secondary hole at the bottom. It should be noted that the aspect ratio of the hole $\left(H_{\mathrm{d}}: D_{\text {in }}\right)$ is significantly lower than implied by this schematic, typically being in the range $0.003-0.032$.

3.1.1. Low frequencies ( $f=1,2$ and $5 \mathrm{~Hz}$ ). For an ejection frequency of $5 \mathrm{~Hz}$ or less, $D_{\text {out }}$ is independent of both frequency and $N_{\mathrm{d}}$, as shown in figure 3(a). The mean values of $D_{\text {out }}$ for the via-holes produced at $1 \mathrm{~Hz}, 2 \mathrm{~Hz}$ and $5 \mathrm{~Hz}$ are calculated
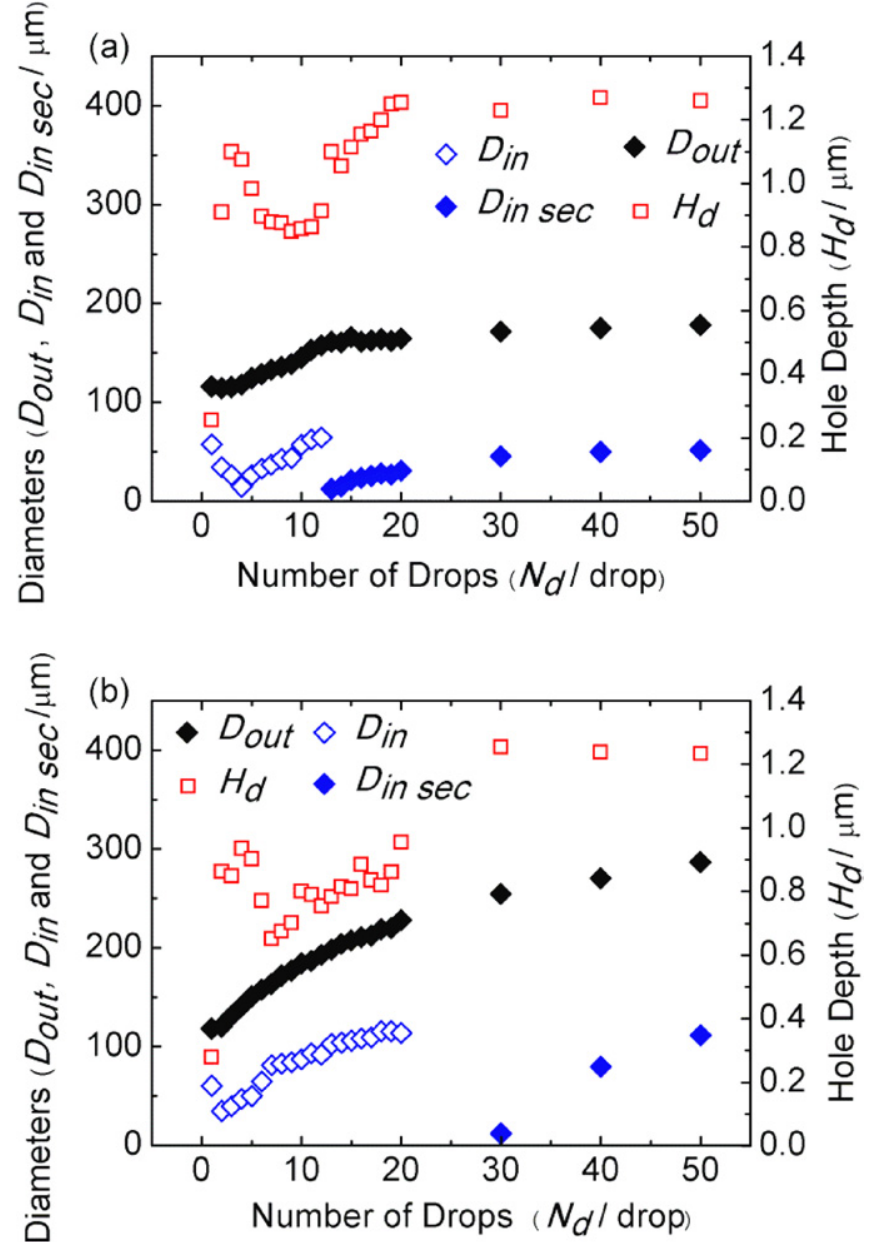

Figure 4. The profile of $D_{\text {out }}, D_{\text {in }}$ and $H_{\mathrm{d}}$ versus $N_{\mathrm{d}}$ at droplet ejection frequencies of $(a) 10 \mathrm{~Hz}$ and $(b) 20 \mathrm{~Hz}$.

to be $122.5 \mu \mathrm{m}, 123.3 \mu \mathrm{m}$ and $120.7 \mu \mathrm{m}$, respectively. When the ejection frequency is maintained at no more than $5 \mathrm{~Hz}$, $D_{\text {in }}$ also remains unchanged throughout the etching process, except for holes created by dispensing only one drop, as shown in figure 3(b).

3.1.2. Medium frequencies ( $f=10$ and $20 \mathrm{~Hz}$ ). Instead of remaining the same, as occurs at low frequencies, $D_{\text {out }}$ of the via-holes formed at $10 \mathrm{~Hz}$ and $20 \mathrm{~Hz}$, as shown by solid black diamonds in figures $4(a)$ and $(b)$, follows a power law relationship with increasing $N_{\mathrm{d}}$, with the exponents being 0.149 and 0.256 , respectively. The volume loss due to evaporation at the frequencies used in the experiments is assumed to be responsible for the deviation in this exponent 


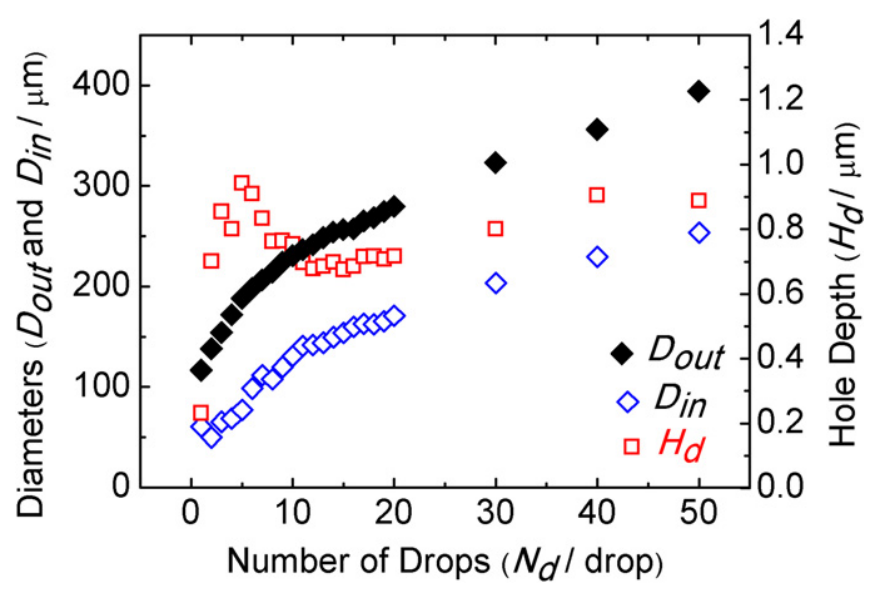

Figure 5. The profile of $D_{\text {out }}, D_{\text {in }}$ and $H_{\mathrm{d}}$ versus $N_{\mathrm{d}}$ at $50 \mathrm{~Hz}$.

from the theoretical value of $1 / 3$ reported by de Gans et al [15]. At such frequencies, $D_{\text {in }}$ also increases with $N_{\mathrm{d}}$, as illustrated by the unfilled blue diamonds in figures $4(a)$ and $(b)$. A new via-hole began to form at the bottom of the initial hole after several drops, which is referred to as a secondary via-hole herein. The profile of a via-hole with a secondary hole is shown in figure $2(b)$ and its development with $N_{\mathrm{d}}$ is represented by solid blue diamonds in figures $4(a)$ and $(b)$. Secondary viaholes began to appear when no less than 13 drops had been dispensed at $10 \mathrm{~Hz}$. However, at $20 \mathrm{~Hz}$ such secondary viaholes were not observed before 20 drops were dispensed, i.e. the appearance of secondary via-holes is delayed by increasing the dispensing frequency.

3.1.3. High frequencies ( $f=50 \mathrm{~Hz}$ ). At a droplet ejection frequency of $50 \mathrm{~Hz}$, the size evolution with $N_{\mathrm{d}}$ resembles that at 10 and $20 \mathrm{~Hz}$, as shown in figure 5 . $D_{\text {out }}$ still follows a power law relationship with increasing $N_{\mathrm{d}}$, with the exponent being 0.307 , closer to the theoretical value of $1 / 3 . D_{\text {in }}$ also increases with $N_{\mathrm{d}}$. However, secondary holes were not found to develop within 50 drops at $50 \mathrm{~Hz}$.

\subsubsection{Mechanism discussion. The size evolution of the} via-holes throughout the etching process is attributed to the interrelationship between the volume of the sessile drop on the poly4(-vinyl phenol) layer and the extent of its evaporation before another drop merges in, which will be discussed in more detail later in this paper.

It has been shown that solvent first diffuses into the polymer during dissolution resulting in a swollen feature, which is then followed by the formation of intermediate layers between pure solvent and pure polymer [28]. Since the polymer layer in this experiment is very thin, it is assumed that chain disentanglement occurs immediately after solvent diffusion. The time scale of the polymer dissolution process can therefore be estimated to be similar to that of the solvent absorption into the polymer. It has been reported by Desie et al [29] that absorption usually happens on the time scale of a few milliseconds, which is much slower than impact and spreading, which are processes that take place within several microseconds. For example, according to
Dong et al [30], the significant stages of $\varnothing 40-50 \mu \mathrm{m}$ droplet impact and sessile drop formation take less than $100 \mu \mathrm{s}$. The optically observable evaporation process of ethyl acetate on poly(ethylmethacrylate) usually takes $0.3 \mathrm{~s}$ for a $50 \mathrm{pl}$ droplet [10]. The evaporation of ethanol (boiling point $78^{\circ} \mathrm{C}$, vapour pressure $9 \mathrm{kPa}$ ) droplets can be assumed to approximate that of ethyl acetate (boiling point $77^{\circ} \mathrm{C}$, vapour pressure $14 \mathrm{kPa}$ ) droplets due to the similarity in their evaporation properties. Therefore the sequence of the time scale for these processes in ascending order accordingly is: droplet impact and spreading on the polymer layer to form a sessile drop; polymer dissolution into the sessile drop; and sessile drop evaporation into the ambient.

As schematically illustrated in figures $6(a)$ and $(b)$, it is postulated that when a single ethanol droplet is jetted onto the poly4(-vinyl phenol) layer, it first impacts on the surface and quickly wets and spreads to form a sessile drop covering an area within the diameter of $\varphi$. This is quickly followed by the dissolution of the polymer into the ethanol drop, which is accompanied by simultaneous ethanol evaporation continuing beyond the completion of the polymer dissolution. Dissolved polymer is transferred outwards from the central area and subsequently re-deposited on the perimeter of the crater-like via-hole due to the coffee stain effect, in which faster evaporation at the perimeter causes more rapid volume loss at the periphery, thereby resulting in a compensating outward flow of material to maintain the sessile droplet shape [31,32]. At low frequencies, i.e. no more than $5 \mathrm{~Hz}$, sufficient time is available for the preceding drop to evaporate substantially prior to the arrival of another drop. The process repeats itself from $\left(c_{1}\right)$ to $\left(e_{1}\right)$ for each droplet jetting, as shown in figure 6 . The contact line of the second sessile drop is believed to be pinned at the same location as for the preceding drop when the next droplet wets the polymer. Therefore $D_{\text {out }}$ remains constant. It is challenging to visualize the process of an ethanol droplet wetting on a soluble substrate due to issues such as fast evaporation and a low contact angle. However, it is helpful to understand the process by analogy with time resolved images of a droplet wetting on a non-soluble substrate. Lim et al presented such images for a water droplet and ethylene glycol droplet impacting and wetting on a silicon substrate at room temperature [33].

No removal or evaporation of the polymer occurs, so the dissolved polymer can only be re-distributed and the hole formation is due to migration of the polymer from the centre of the via-hole to the perimeter during ethanol evaporation. The occupancy of space in the via-hole by re-precipitated polymer leads to a decrease in $D_{\text {in }}$ as the via-hole is etched deeper. Dispensing more ethanol drops results in more polymer dissolution and re-deposition until the bottom is fully revealed free of residual polymer. When the substrate at the bottom of the via-hole is exposed, no more polymer is available to be transferred from the centre to the periphery of the viahole. Consequently $D_{\text {in }}$ remains constant. This explains the dependence of $D_{\text {in }}$ on the thickness of the polymer layer as reported by Kawase et al [14].

At an ejection frequency of $50 \mathrm{~Hz}$, the time interval between two consecutive dispensed drops is insufficient for 


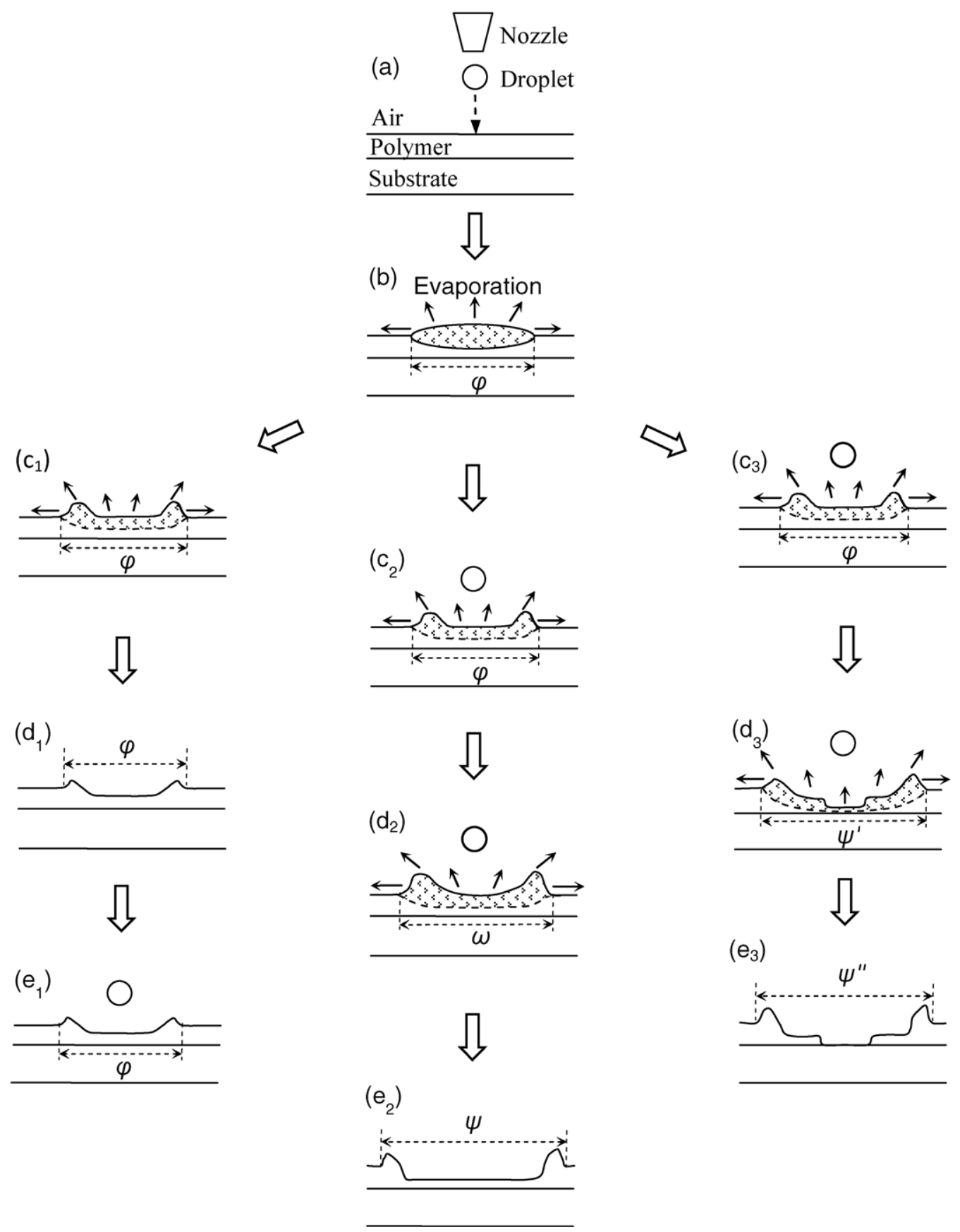

Figure 6. The process of via-hole etching at different ejection frequencies: $(a)$ Initial configuration; $(b)$ After impact and spreading of the first drop; $\left(c_{1}\right)-\left(e_{1}\right)$. The process of etching at frequencies sufficiently low for the preceding drop to evaporate before another drop impacts; $\left(c_{2}\right)-\left(e_{2}\right)$. The process of etching at frequencies significantly above the threshold frequency featuring contact line enlargement and no secondary via-hole; $\left(c_{3}\right)-\left(e_{3}\right)$. The process of etching at frequencies higher than the threshold frequency featuring contact line enlargement and a secondary via-hole.

the sessile drop to substantially evaporate before the next drop merges into it. Incomplete evaporation results in continuous volume expansion of the sessile drop due to the merging of multiple drops. This transforms the process from drops impacting solid, as occurs at low ejection frequencies, to drops impacting liquid. Accordingly, the contact line moves outwards, rather than being pinned locally, which enlarges both $D_{\text {out }}$ and $D_{\text {in }}$ in consequence. Even though a drop can penetrate the shallow liquid film, the low viscosity of the liquid film allows it to flow back and recover after the collision. Therefore no secondary via-hole is observed within 50 drops at $50 \mathrm{~Hz}$.
This process is illustrated in figures $6\left(c_{2}\right)-\left(e_{2}\right)$. Nonetheless, due to a deeper penetration by subsequent ethanol drops, re-deposited polymer still leads initially to a $D_{\text {in }}$ decrease. Then $D_{\text {in }}$ starts to increase as a result of the dominating effect of contact line enlargement.

It is thought that the hole formation process can still be considered as drops impacting liquid at $10 \mathrm{~Hz}$ and $20 \mathrm{~Hz}$. However, the viscosity of the liquid film can be expected to be higher than at $50 \mathrm{~Hz}$, due to less accumulation of incompletely evaporated solvent. It is believed that an appropriate impact velocity can provide sufficient kinetic energy transfer to 

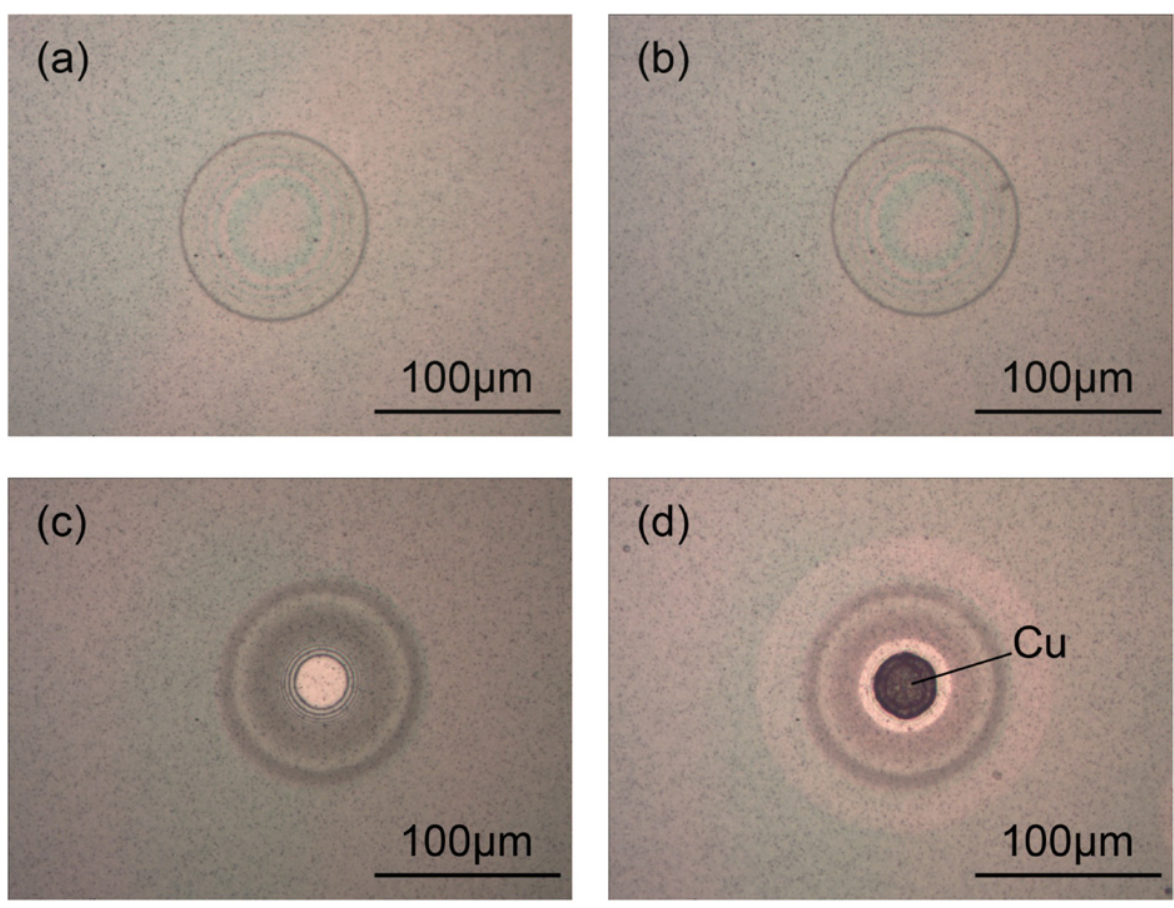

Figure 7. Comparison of via-holes produced at $1 \mathrm{~Hz}$ before and after electroplating. (a) The via-hole produced by 2 drops before electroplating. $(b)$ The via-hole produced by 2 drops after electroplating. ( $c$ ) The via-hole produced by 15 drops before electroplating. (d) The via-hole produced by 15 drops after electroplating.

counter the flowing back of the viscous liquid. If the liquid film cannot then fully recover, due to its relatively high viscosity, this will contribute to the formation of a secondary via-hole, as illustrated in figures $6\left(c_{3}\right)-\left(e_{3}\right)$.

\subsection{Use of electroplating as a penetration test}

Electroplating was utilized to test the completeness of penetration of via-holes produced at low frequencies. Prior to spin coating poly4(-vinyl phenol) onto the sample, a thin layer of sputtered $\mathrm{Cu}$ was applied to the substrate, which was used as a conductive seed-layer for electroplating. A commercially available copper sulfate based plating solution was used as the electrolyte. Because poly4(-vinyl phenol) is an insulating material, $\mathrm{Cu}$ can only be electroplated in the viahole if it is completely etched through to the bottom so that the $\mathrm{Cu}$ layer is exposed to the electrolyte. An area where there is no deposition of $\mathrm{Cu}$ in the via-hole therefore indicates the presence of polymer residue. Dispensing frequencies of 1 and $2 \mathrm{~Hz}$ were used to produce via-holes for this penetration test. The sample could be used for electroplating without a crosslinking step, due to the insolubility of the as-deposited polymer in water. Electroplated $\mathrm{Cu}$ was observed in the viaholes produced by seven drops and more, but no $\mathrm{Cu}$ was seen in via-holes produced by less than seven drops. Figure 7 shows optical microscopy photos of partially etched and completely etched via-holes before and after electroplating at $1 \mathrm{~Hz}$.

\subsection{Threshold frequency}

Based on the experiments and analysis above, we conclude that a threshold dispensing frequency exists for an ethanol drop of a given volume, under which no via-hole enlargement will occur. This threshold frequency, $f_{\mathrm{c}}$, is defined here as the droplet ejection frequency for a given droplet volume under which both $D_{\text {out }}$ and $D_{\text {in }}$ become constant once a sufficient number of droplets has been dispensed assuming that the polymer layer is completely penetrated. An experiment was therefore performed to investigate $f_{\mathrm{c}}$.

For this experiment, a $7.8 \mathrm{wt} \%$ polymer solution was used with the same spin coating parameters to obtain a thinner layer of polymer, i.e. approximately $0.77 \mu \mathrm{m}$ thick. The droplet size was reduced through further adjustment of the jetting waveform, resulting in a droplet diameter and volume estimated to be $33 \mu \mathrm{m}$ and $18.8 \mathrm{pl}$, respectively. Since the size development of the via-holes with increasing $N_{\mathrm{d}}$ has already been demonstrated in section 3.1, only 7 via-holes were produced using 1, 2, 7, 10, 12 and 15 drops, respectively, for each frequency. Figure 8 shows the results for these experiments.

As illustrated in figure 8(a), no via-hole enlargement is observed for dispensing frequencies under $16 \mathrm{~Hz}$, while the outer diameter starts to increase as more drops are dispensed at $16 \mathrm{~Hz}$. The via-hole depth also decreases with an increasing number of drops at $16 \mathrm{~Hz}$, which indicates incomplete penetration, i.e. $f_{\mathrm{c}}$ was found to be $15 \mathrm{~Hz}$. Even though $D_{\text {in }}$ levels off to a constant value after dispensing of multiple droplets at ejection frequencies no more than $15 \mathrm{~Hz}$, $D_{\text {in }}$ varies according to the specific frequency used, as depicted in figure $8(b)$. This variation is attributed to the liquid flowing back towards the centre.

It is believed that the wetting of the sessile drop follows a constant contact area mode when the dispensing frequency is lower than $f_{c}$. Subsequent drops merge together without 

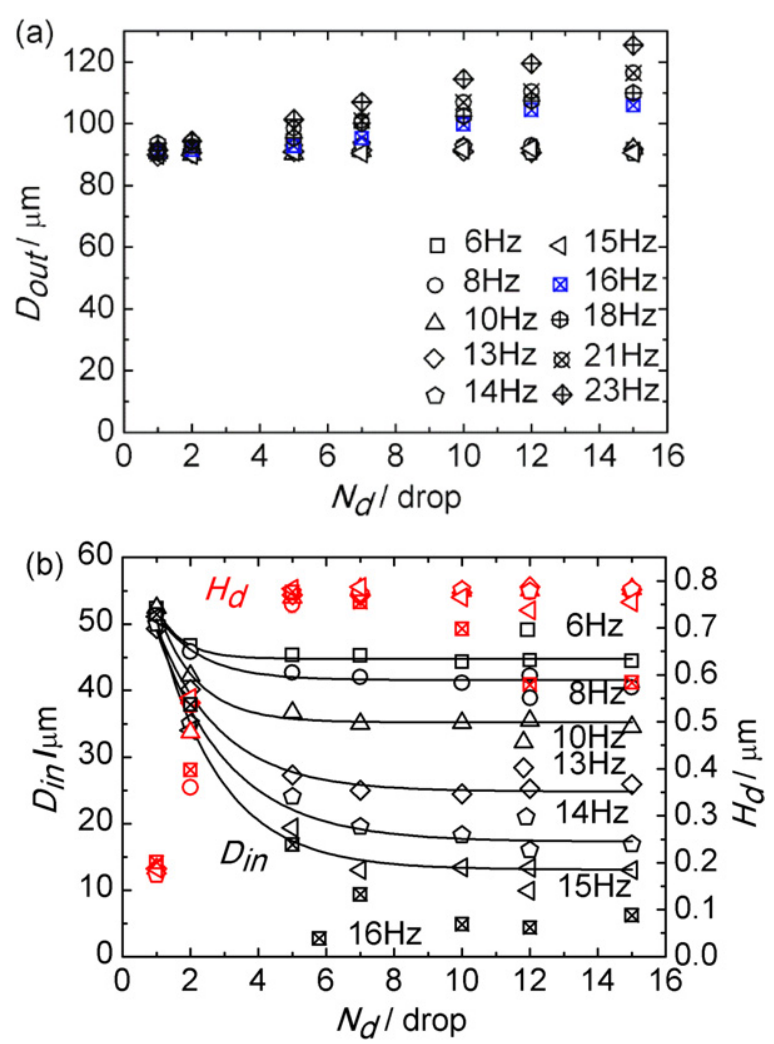

Figure 8. The profile of (a) $D_{\text {out }}$ and (b) $D_{\text {in }}$ and $H_{\mathrm{d}}$ versus $N_{\mathrm{d}}$ at different droplet ejection frequencies.

enlarging the contact area, due to the locally pinned contact line. This constant contact area mode contributes to $D_{\text {out }}$ remaining constant, as illustrated in figures $6\left(c_{1}\right)-\left(e_{1}\right)$. At such dispensing frequencies, there is insufficient time available for each new drop to evaporate, and the increasing amount of solvent merging into the sessile drop results in a decrease in its viscosity. The pinned contact line and the decreasing viscosity of the sessile drop facilitate the dissolved polymer flowing back into the centre. This inward micro-fluidic flow counters the outward flow caused by the coffee stain effect during solvent evaporation. The net flow, which is still outwards, determines $D_{\text {in }}$. Therefore the higher the dispensing frequency is, the lower is the viscosity of the sessile drop, and the further it can flow back into the bottom centre.

As the frequency rises above $f_{c}$, further increases in the volume of the sessile drop and the kinetic energy transfer break the equilibrium for the constant contact area mode. The sessile drop then expands its contact area with the polymer surface until reaching a new equilibrium. This, in turn, contributes to the increase in $D_{\text {out }}$. Meanwhile, the low viscosity of the sessile drop helps the liquid recover after impact, leading to a decrease in the via-hole depth, as shown by the crossed red squares in figure $8(b)$, which indicates incomplete penetration of the polymer.

\subsection{Incomplete penetration}

Unlike traditional etching techniques, there is no reaction during the inkjet via-hole etching process, only polymer dissolution and re-deposition. Accordingly, even if the

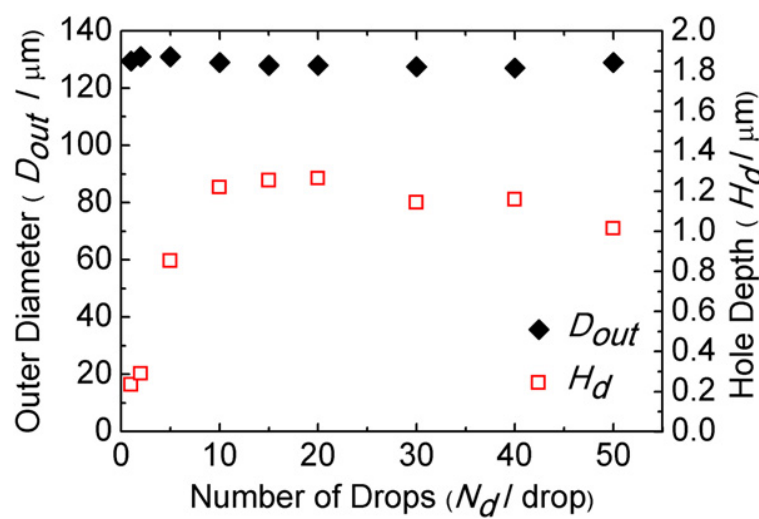

Figure 9. The profile of $D_{\text {out }}$ and $H_{\mathrm{d}}$ versus $N_{\mathrm{d}}$ with $120 \mathrm{~s}$ jetting delay for deionized water.

polymer can be dissolved and transferred to the periphery during solvent evaporation, it can neither escape into the ambient with the solvent nor be washed away after etching by other solvents, but only remain in the via-hole occupying some space. It is postulated that this is the cause of the penetration limit. The more polymer the etchant dissolves, the more space is needed in the hole simultaneously to accommodate the dissolved polymer. Therefore it can be assumed that the polymer layer cannot be completely penetrated when it reaches a certain thickness.

To test this, a different water-soluble polymer of polyacrylamide was used to produce a thicker dielectric layer on top of the glass substrate. The solution was spin coated onto the substrate at $2500 \mathrm{rpm}$ for $30 \mathrm{~s}$. The induced polymer thickness was measured to be about $6 \mu \mathrm{m}$.

In this case deionized water was used as the etchant. The droplet ejection frequency was set to $1 \mathrm{~Hz}$ and a script was written to delay each jetting for $120 \mathrm{~s}$ to give each droplet substantial time to evaporate. The jetting parameters were set to $t_{\text {rise }}=t_{\text {fall }}=3 \mu \mathrm{s}, t_{\text {dwell }}=27 \mu \mathrm{s}$ and $U_{\text {dwell }}=23 \mathrm{~V}$. The resulting droplet diameter was estimated to be $48.14 \mu \mathrm{m}$. Figure 9 illustrates the evolution of $D_{\text {out }}$ and $H_{\mathrm{d}}$ with $N_{\mathrm{d}}$ with a jetting delay of $120 \mathrm{~s}$ for deionized water. It can be seen from figure 9 that $D_{\text {out }}$ stays constant while $H_{\mathrm{d}}$ is well below the thickness of the polymer, which indicates incomplete penetration.

\section{Conclusion}

The impact of droplet ejection frequency on the dimensions of the via-hole produced versus the number of drops was investigated. The effect of droplet ejection frequency is directly a consequence of the resulting time available for the sessile drop to evaporate before another droplet is jetted and therefore whether complete evaporation occurs or solvent accumulates. At frequencies below the threshold frequency $f_{\mathrm{c}}$, when the evaporation time is sufficient, the size of viaholes produced is independent of the number of drops used to etch them. Therefore, dispensing more drops to remove the polymer residue at the bottom will not enlarge the via-hole. At frequencies above $f_{\mathrm{c}}$, the size of via-hole will increase with the number of drops dispensed. The threshold frequency is 
defined here as the ejection frequency below which both $D_{\text {out }}$ and $D_{\text {in }}$ stay constant and independent of $N_{\mathrm{d}}$ once the layer is completely etched through. For a droplet volume of $18.8 \mathrm{pl}$, $f_{\mathrm{c}}$ has been found experimentally to be $15 \mathrm{~Hz}$. A secondary via-hole can be found at the bottom of the initial via-hole. This is believed to be because the higher viscosity of the liquid film makes it slow to recover its equilibrium shape after impact. Electroplating was used to identify the presence of any residual polymer at the bottom of via-holes. Via-holes produced by seven drops or more show no presence of residue based on the fact that $\mathrm{Cu}$ deposition occurred over the whole area of the hole.

Using inkjet etching to locally dissolve via-holes is an interestingly new data driven technique for printed electronics. However, there are disadvantages and shortcomings. It requires highly repeatable drop production with little trajectory deviation to deliver high quality via-hole creation. Solvents that can readily dissolve relevant dielectric polymers and which rapidly evaporate should be used as the etchant. Since material is re-deposited without being removed, the via-hole refilling issue limits the penetration depth, which makes it extremely difficult to produce high aspect ratio holes. Significant further research needs to be done before this technique's applicability within industrial applications can be fully evaluated, but it shows promise for a number of potential applications.

\section{References}

[1] de Gans B J, Duineveld P and Schubert U 2004 Adv. Mater. 16203

[2] Gamerith S, Klug A, Scheiber H, Scherf U, Moderegger E and List E 2007 Adv. Funct. Mater. 173111

[3] Mengel M and Nikitin I 2010 Microelectron. Eng. 87593

[4] Perelaer J, Hendriks C E, de Laat A W M and Schubert U S 2009 Nanotechnology 20165303

[5] Sele C, von Werne T, Friend R and Sirringhaus H $2005 A d v$. Mater. 17997

[6] Sirringhaus H, Kawase T, Friend R H, Shimoda T, Inbasekaran M, Wu W and Woo E P 2000 Science 2902123

[7] Bonaccurso E, Butt H J, Hankeln B, Niesenhaus B and Graf K 2005 Appl. Phys. Lett. 86124101

[8] Pericet-Camara R, Best A, Nett S K, Gutmann J S and Bonaccurso E 2007 Opt. Express 159877

[9] Xia Y J and Friend R H 2007 Appl. Phys. Lett. 90253513

[10] Li G F, Graf K, Bonaccurso E, Golovko D S, Best A and Butt H J 2007 Macromol. Chem. Phys. 2082134

[11] Karabasheva S, Baluschev S and Graf K 2006 Appl. Phys. Lett. 89031110
[12] Grimaldi I A, De Girolamo Del Mauro A, Loffredo F, Nenna G, Villani F and Minarini C 2011 Proc. SPIE Optical Meaurement Systems for Industrial Inspection VII (Munich, Germany, 23-26 May 2011) (Poster Session: Nondestructive Testing and Process Monitoring vol 8082) ed P H Lehmann et al pp 808244-1-808244-7

[13] Grimaldi I A, De Girolamo Del Mauro A, Nenna G, Loffredo F, Minarini C and Villani F 2010 AIP Conf. Proc. $V$ Int. Conf. on Times of Polymers (TOP) and Composites (Ischia, Italy, 20-23 June 2010) (vol 1255) ed A D'Amore et al pp 104-6

[14] Kawase T, Sirringhaus H, Friend R H and Shimoda T 2001 Adv. Mater. 131601

[15] de Gans B J, Hoeppener S and Schubert U S 2007 J. Mater. Chem. 173045

[16] Shmuylovich L, Shen A Q and Stone H A 2002 Langmuir 183441

[17] Kajiya T, Monteux C, Narita T, Lequeux F and Doi M 2009 Langmuir 256934

[18] Pericet-Camara R, Bonaccurso E and Graf K 2008 ChemPhysChem 91738

[19] Shen X, Ho C-M and Wong T-S 2010 J. Phys. Chem. B 1145269

[20] Hu H and Larson R G 2006 J. Phys. Chem. B 1107090

[21] Chon C H, Paik S, Tipton J B Jr and Kihm K D 2007 Langmuir 232953

[22] Poulard C and Damman P 2007 Europhys. Lett. 8064001

[23] Grimaldi I A, De Girolamo Del Mauro A, Nenna G, Loffredo F, Minarini C and villani F $2011 \mathrm{~J}$. Appl. Polym. Sci. 1223637

[24] de Gans B J, Hoeppener S and Schubert U S 2006 Adv. Mater. 18910

[25] Xia Y J and Friend R H 2006 Appl. Phys. Lett. 88163508

[26] Gan H Y, Shan X, Eriksson T, Lok B K and Lam Y C 2009 J. Micromech. Microeng. 19055010

[27] Sakai S 2000 NIP16: Int. Conf. on Digital Printing Technologies (Vancouver, 15-20 October 2000) (vol 16) pp 15-20

[28] Miller-Chou B A and Koenig J L 2003 Prog. Polym. Sci. 281223

[29] Desie G, Allaman S, Lievens O, Anthonidssen K and Soucemarianadin A 2002 NIP18: Int. Conf. on Digital Printing Technologies (San Diego, CA, 29 September-4 October 2002) (vol 18) pp 360-5

[30] Dong H, Carr W W and Morris J F 2006 Rev. Sci. Instrum. 77085101

[31] Deegan R D, Bakajin O, Dupont T F, Huber G, Nagel S R and Witten T A 1997 Nature 389827

[32] Deegan R D, Bakajin O, Dupont T F, Huber G, Nagel S R and Witten T A 2000 Phys. Rev. E 62756

[33] Lim T, Han S, Chung J, Chung J T, Ko S and Grigoropoulos C P 2009 Int. J. Heat Mass Transfer 52431 